\title{
Stents Farmacológicos Eluidores de Everolimus na Prática no Mundo Real
}

\author{
Ver artigo relacionado \\ na página 357
}

\author{
Bimmer E. Claessen 1, Roxana Mehran ${ }^{2,3}$, \\ Gregg W. Stone ${ }^{2,4}$
}

$\mathbf{E}$ m dezembro de 1999 ocorreu o primeiro implante do que seria depois o primeiro stent farmacológico disponível comercialmente. ${ }^{1}$ Uma metanálise com ensaios clínicos randomizados, que comparou a primeira geração de stents farmacológicos eluidores de sirolimus e de paclitaxel com stents metálicos convencionais, mostrou redução da necessidade de revascularização da lesão-alvo de cerca de $60 \%$ a $70 \%$ em todos os tipos de lesão. ${ }^{2}$ Entretanto, o risco de trombose muito tardia do stent ( $>1$ ano) foi maior com esses stents farmacológicos de primeira geração ${ }^{3}$, indicando claramente a necessidade de um stent que oferecesse resultados com maior segurança sem comprometer a excelente eficácia, em termos de redução da reestenose.

O stent eluidor de everolimus (SEE) $X$ ience $^{\text {TM }} \mathrm{V}$ (Abbot Vascular, Santa Clara, Estados Unidos, também distribuído como Promus ${ }^{\mathrm{TM}}$, Boston Scientific, Natick, Estados Unidos) é um stent farmacológico de segunda geração. Consiste de uma plataforma de cromo-cobalto, de hastes finas $(81,3 \mu \mathrm{m})$, coberta por um fluoropolímero que libera um análogo da rapamicina, o everolimus, fármaco com propriedades anti-inflamatórias e citostáticas. Um número relativamente elevado de ensaios randomizados controlados foi publicado, comparando o SEE com uma variedade de outros stents farmacológicos. Nesses estudos, o SEE foi comprovadamente superior aos stents farmacológicos de primeira geração eluidores de paclitaxel, tanto em termos de segurança como de eficácia. ${ }^{4,5}$ Mais ainda, o SEE é pelo menos tão eficaz e seguro quanto o stent farmacológico de primeira geração liberador de sirolimus e o stent farmacológico de segunda geração liberador de zotarolimus. ${ }^{6-9}$

Uma das limitações dos ensaios randomizados reside no fato de que seus resultados, caracteristica- mente, se aplicam apenas aos pacientes com critérios específicos de inclusão e de exclusão. É interessante notar que uma publicação recente sugeriu que isso também se estende aos ensaios randomizados controlados, que usam um delineamento que inclui todos os pacientes (all-comers). ${ }^{10}$ No ensaio all-comers RESOLUTE, apenas metade dos all-comers foi inscrita, demonstrando-se que os pacientes que não participaram do estudo tiveram resultados clínicos piores. Portanto, os registros clínicos de alta qualidade desempenham papel importante, no sentido de proporcionar uma perspectiva complementar aos ensaios randomizados controlados.

Os resultados do Brazil Xience V Everolimus-Eluting Coronary Stent System 'real world' outcomes registry (registro BRAVO), publicado nesta edição da Revista Brasileira de Cardiologia Invasiva ${ }^{11}$, acrescenta a nosso conhecimento dados a respeito da eficácia e da segurança do SEE na prática clínica diária. Nesse registro multicêntrico, são apresentados os resultados em 6 meses de 535 pacientes com lesões coronárias nativas, tratados com SEE em 25 centros. Essa foi uma coorte relativamente complexa, em que aproximadamente $70 \%$ dos pacientes apresentavam lesões classe B2/C, segundo a classificação do American College of Cardiology/American Heart Association (ACC/AHA), 41,9\% dos quais apresentavam síndromes coronárias agudas e comprimento médio da lesão de 17,8 mm.

No registro BRAVO, o SEE demonstrou excelente eficácia clínica e segurança aos 6 meses, com taxa de 4,3\% de eventos cardíacos adversos maiores (ECAM), compostos por morte cardíaca, infarto do miocárdio (IM) e revascularização do vaso-alvo (RVA), e taxa de $0,75 \%$ de trombose do stent definitiva/provável/possível, de acordo com os critérios do Academic Research Consortium (ARC). ${ }^{12}$ As taxas de morte cardíaca, IM e RVA

\footnotetext{
1 Department of Cardiology - Academic Medical Center - University of Amsterdam - Amsterdã, Holanda.

2 Cardiovascular Research Foundation - New York, NY, Estados Unidos.

3 Department of Cardiology - Mount Sinai Medical Center - New York, NY, Estados Unidos.

4 Department of Medicine - Division of Cardiology - Columbia University Medical Center - New York, NY, Estados Unidos.

Correspondência: Bimmer E. Claessen. Department of Cardiology - Academic Medical Center - University of Amsterdam - Meibergdreef 9 1105 AZ - Amsterdam, the Netherlands.

E-mail: b.e.claessen@amc.uva.nl

Recebido em: 12/12/2011 • Aceito em: 13/12/2011
} 
aos 6 meses foram de, respectivamente, 1,1\%, 2,2\% e $1,3 \%$. Isso está de acordo com relatos anteriores de registros realizados em Roterdã e Milão. ${ }^{13,14}$

Integrando os principais resultados de ensaios randomizados controlados, as análises post-hoc (exploratórias) de ensaios controlados randomizados e os resultados de registros pós-comercialização, tais como o registro BRAVO, indicam eficácia e segurança favoráveis do perfil do dispositivo SEE, mesmo em uso off-label (uso em indicação que não consta da bula) no mundo real. No entanto, o desenho do stent continua a evoluir e novas gerações de stents coronários, inclusive de stents farmacológicos com polímeros biodegradáveis e plataformas completamente bioabsorvíveis, estão sendo desenvolvidas e passando por ensaios fases II e III.

Conforme o excelente desempenho clínico demonstrado no registro BRAVO, o SEE é uma escolha adequada para lesões complexas na prática clínica diária e deve ser o dispositivo de comparação em ensaios clínicos randomizados e controlados que investiguem a próxima geração de stents coronários. Na verdade, os resultados com o SEE podem ser difíceis de superar.

\section{CONFLITO DE INTERESSES}

Bimmer E. Claessen declara não haver conflito de interesses relacionado a este manuscrito. Roxana Mehran recebeu financiamento para pesquisa da Bristol-Myers Squibb/Sanofi e é consultora da Regado Biosciences, Astra Zeneca, Abbott Vascular e Janssen Pharmaceuticals. Gregg W. Stone é consultor da Abbott Vascular, Medtronic e Boston Scientific.

\section{REFERÊNCIAS}

1. Sousa JE, Costa JR Jr, Abizaid AS. 10-year follow-up of the first Cypher stent implanted in human: an invasive evaluation with angiography, intravascular ultrasound, and optical coherence tomography. JACC Cardiovasc Interv. 2010;3(5):556-8.

2. Stettler C, Wandel S, Allemann S, Kastrati A, Morice MC, Schömig A, et al. Outcomes associated with drug-eluting and bare-metal stents: a collaborative network meta-analysis. Lancet. 2007;370(9591):937-48.

3. Stone GW, Moses JW, Ellis SG, Schofer J, Dawkins KD, Morice MC, et al. Safety and efficacy of sirolimus- and paclitaxel-eluting coronary stents. N Engl J Med. 2007;356(10): 998-1008.
4. Kedhi E, Joesoef KS, McFadden E, Wassing J, van Mieghem C, Goedhart D, et al. Second-generation everolimus-eluting and paclitaxel-eluting stents in real-life practice (COMPARE): a randomised trial. Lancet. 2010;375(9710):201-9.

5. Stone GW. SPRIT IV: a prospective randomized trial of everolimus-eluting vs. paclitaxel-eluting stents [abstract]. Presented 23/09/2009 at TCT, San Francisco 9 A.D.

6. Kim WJ, Lee SW, Park SW, Kim YH, Yun SC, Lee JY, et al. Randomized comparison of everolimus-eluting stent versus sirolimus-eluting stent implantation for de novo coronary artery disease in patients with diabetes mellitus (ESSENCEDIABETES): results from the ESSENCE-DIABETES trial. Circulation. 2011;124(8):886-92.

7. Serruys PW, Silber S, Garg S, van Geuns RJ, Richardt G, Buszman PE, et al. Comparison of zotarolimus-eluting and everolimus-eluting coronary stents. N Engl J Med. 2010;363(2): 136-46.

8. Park DW, Kim YH, Song HG, Ahn JM, Kim WJ, Lee JY, et al. Comparison of everolimus- and sirolimus-eluting stents in patients with long coronary artery lesions: a randomized LONG-DES-III (Percutaneous Treatment of LONG Native Coronary Lesions With Drug-Eluting Stent-III) Trial. JACC Cardiovasc Interv. 2011;4(10):1096-103.

9. Park KW, Chae IH, Lim DS, Han KR, Yang HM, Lee HY, et al. Everolimus-eluting versus sirolimus-eluting stents in patients undergoing percutaneous coronary intervention: the EXCELLENT (Efficacy of Xience/Promus Versus Cypher to Reduce Late Loss After Stenting) randomized trial. J Am Coll Cardiol. 2011; 58(18):1844-54

10. de Boer SP, Lenzen MJ, Oemrawsingh RM, Simsek C, Duckers HJ, van der Giessen WJ, et al. Evaluating the 'all-comers' design: a comparison of participants in two 'all-comers' $\mathrm{PCl}$ trials with non-participants. Eur Heart J. 2011;32(17):2161-7.

11. Abreu-Silva EO, Costa RA, Abizaid AS, Perin M, Cardoso RF, Prudente ML, et al. Stents farmacológicos liberadores de everolimus $\mathrm{Xience}^{\mathrm{TM}} \mathrm{V}$ no tratamento de pacientes com lesões coronárias complexas na prática diária: resultados iniciais do Registro Brasileiro BRAVO. Rev Bras Cardiol Invasiva. 2011; 19(4):357-66.

12. Cutlip DE, Windecker $S$, Mehran R, Boam A, Cohen DJ, van Es GA, et al. Clinical end points in coronary stent trials: a case for standardized definitions. Circulation. 2007;115(17): 2344-51.

13. Onuma Y, Kukreja N, Piazza N, Eindhoven J, Girasis C, Schenkeveld L, et al. The everolimus-eluting stent in realworld patients: 6-month follow-up of the X-SEARCH (Xience V Stent Evaluated at Rotterdam Cardiac Hospital) registry. J Am Coll Cardiol. 2009;54(3):269-76.

14. Latib A, Ferri L, lelasi A, Godino C, Chieffo A, Magni V, et al. Clinical outcomes after unrestricted implantation of everolimus-eluting stents. JACC Cardiovasc Interv. 2009;2(12): 1219-26. 